\title{
OVERLAP OF QRPA STATES BASED ON GROUND STATES OF DIFFERENT NUCLEI*
}

\author{
J. TERASAKI
}

\author{
Division of Physics and Center for Computational Sciences \\ University of Tsukuba, Tsukuba, 305-8577, Japan
}

(Received December 10, 2012)

\begin{abstract}
We show the formulation and the result of test calculation of the overlap of excites states in the quasiparticle random-phase approximation for calculating the nuclear matrix elements of the neutrino-less double beta decay. Our method uses the ground states of the QRPA explicitly. The feasibility of that calculation is demonstrated, and the effectiveness of the truncation used in our method is shown.
\end{abstract}

DOI:10.5506/APhysPolB.44.259

PACS numbers: 21.60.Jz, 23.40.Hc

\section{Introduction}

One of few methods to determine the neutrino mass is to measure the half life (transition rate) of the neutrino-less double beta $(0 \nu \beta \beta)$ decay and to calculate the transition matrix element of that decay (nuclear matrix element), see e.g. Ref. [1]. The half life of the initial state is inversely proportional to the squared nuclear matrix element, and the squared effecitve neutrino mass is included in the inversely-proportional coefficient. The theoretical methods used so far for calculating the nuclear matrix elements are shell model calculation, proton-neutron quasiparticle random-phase approximation ( $p n$-QRPA), projected Hartree-Fock-Bogoliubov (HFB) approximation, interacting boson model, and generator coordinate method (see references in [2]). It is known that the results of these calculations do not converge; in particular, the shell model calculations and the $p n$-QRPA have a tendency of the difference of a factor of 2 [3].

This study is the first step of a new method to calculate the nuclear matrix element. First, we use like-particle QRPA (hereafter we call this approximation simply QRPA) for getting the intermediate nuclear states.

\footnotetext{
* Presented at the Zakopane Conference on Nuclear Physics "Extremes of the Nuclear
} Landscape", Zakopane, Poland, August 27-September 2, 2012. 
This approach is based on the fact that the closure approximation [1] is good in the $0 \nu \beta \beta$ decay. With this approximation and the closure property, it is possible to write the nuclear matrix element in the form of a matrix element of a two-body operator without the intermediate states. Subsequently, we can rearrange the order of the neutron annihilation and the proton creation operators and insert another closure relation with the intermediate states having $(Z+2, N)$ or $(Z, N-2)$. [The initial (parent) nuclear state has $(Z, N)$.] In the application of the QRPA, this closure relation is replaced by the product of the two closure relations obtained based on the initial and the final states, and the overlap of the QRPA states arises. One of advantages of the QRPA is that the anharmonicity is small in many nuclei except for the transition regions between spherical and deformed nuclei. Another advantage is that odd-odd nuclei are not involved, so that it is not necessary to consider whether or not the last odd particle is coupled to the rest of the nucleus weakly.

Another new point of our method is to calculate the overlap of the intermediate QRPA states as rigorously as possible, as explained in this paper; this calculation is more advanced than other calculations performed so far (see Ref. [4] and references therein).

In Sec. 2, we show the formulation of the overlap of the QRPA states and the result of the test calculation briefly. A few results of the calculation are published in Ref. [5], and the detail of the calculation is found in Ref. [2]

\section{Formulation and calculation}

We introduce creation operator of the QRPA state

$$
O_{m}^{I \dagger}=\sum_{\mu<\nu}\left(X_{\mu \nu}^{I m} a_{\mu}^{I \dagger} a_{\nu}^{I \dagger}-Y_{-\mu-\nu}^{I m} a_{-\nu}^{I} a_{-\mu}^{I}\right)
$$

where $a_{\mu}^{I \dagger}$ and $a_{\mu}^{I}$ are the creation and the annihilation operators of the quasiparticle, respectively, associated with the initial ground state (indicated by $I$ ) of the $0 \nu \beta \beta$ decay, and $X_{\mu \nu}^{I m}$ and $Y_{-\mu-\nu}^{I m}$ stand for the forward and the backward amplitudes of the QRPA solution $m$, respectively. The quasiparticle states are ordered, and $-\mu$ expresses that its $z$-component of the angular momentum $K_{-\mu}$ is equal to $-K_{\mu}$. The axial and the parity symmetries of the nuclear and the quasiparticle states are assumed. We have similar equations for the final state of the decay (suffix $F$ instead of $I$ ). The initial QRPA ground state $|I\rangle$ is defined by $O_{m}^{I}|I\rangle=0$ and this ground state has a relation [6] to the HFB ground state $|i\rangle$ as 


$$
\begin{aligned}
|I\rangle & =\frac{1}{\mathcal{N}_{I}} \prod_{K \pi} \exp \left[v_{I}^{(K \pi)}\right]|i\rangle, \\
v_{I}^{(K \pi)} & =\sum_{\mu \nu \mu^{\prime} \nu^{\prime}} \frac{1}{1+\delta_{K 0}}\left(Y_{I} \frac{1}{X_{I}}\right)_{\mu \nu, \mu^{\prime} \nu^{\prime}}^{\dagger} a_{\mu}^{I \dagger} a_{\nu}^{I \dagger} a_{\mu^{\prime}}^{I \dagger} a_{\nu^{\prime}}^{I \dagger} .
\end{aligned}
$$

$K$ and $\pi$ denote the $z$-component of the angular momentum and the parity of the QRPA solutions, respectively; in principle, all $(K \pi)$ are used. $\mathcal{N}_{I}$ is the normalization factor. The quasi boson approximation is used in deriving the last equation, and in relation to this approximation, we have $K_{\mu}+K_{\nu}=$ $-K_{\mu^{\prime}}-K_{\nu^{\prime}}=K$. The parity of two quasiparticle states $\mu \nu$ as well as $\mu^{\prime} \nu^{\prime}$ is equal to $\pi$. A matrix expression of the forward and backward amplitudes is used in Eq. (1). Using an expansion-truncation of $|I\rangle$ and $|F\rangle$ up to the first order with respect to $v_{I}^{(K \pi)}$ and $v_{F}^{(K \pi)}$, respectively, we can write the overlap of the QRPA states as

$$
\begin{aligned}
\left\langle F\left|O_{m}^{F} O_{m^{\prime}}^{I \dagger}\right| I\right\rangle \simeq & \frac{1}{\mathcal{N}_{I} \mathcal{N}_{F}}\left\{\left\langle f\left|O_{m}^{F} O_{m^{\prime}}^{I \dagger}\right| i\right\rangle+\sum_{K \pi}\left(\left\langle f\left|v_{F}^{(K \pi) \dagger} O_{m}^{F} O_{m^{\prime}}^{I \dagger}\right| i\right\rangle\right.\right. \\
& \left.\left.+\left\langle f\left|O_{m}^{F} O_{m^{\prime}}^{I \dagger} v_{I}^{(K \pi)}\right| i\right\rangle+\left\langle f\left|v_{F}^{(K \pi) \dagger} O_{m}^{F} O_{m^{\prime}}^{I \dagger} v_{I}^{(K \pi)}\right| i\right\rangle\right)\right\},
\end{aligned}
$$

where $|f\rangle$ is the final HFB ground state, and $\mathcal{N}_{I}^{2}\left(\mathcal{N}_{F}^{2}\right)$ is calculated up to the fouth order with respect to $v_{I}^{(K \pi)}\left(v_{F}^{(K \pi)}\right)$.

The numerical result is shown in Fig. 1. This calculation was performed for ${ }^{26} \mathrm{Mg}$ and ${ }^{26} \mathrm{Si}$ (virtual initial and final states) with the Skyrme energy density functional $\mathrm{SkM}^{*}$ [7] and the volume pairing energy density functional [8]. (For detail, see Ref. [2].) The QRPA states of $(K \pi)=(0+)$ are used. It is seen that the convergence is obtained at the first order with respect to $v_{I}^{(K \pi)}$ or $v_{F}^{(K \pi)}$ [that is up to the third term of Eq. (2)]. We found two more important results; $(K \pi)$ used in the summation of Eq. (2) different from that of the QRPA state $\left(K_{m} \pi_{m}\right)$ have little contribution to the overlap except for the spurious states $(m=1$ and 2 in Fig. 1). [Those $(K \pi)$ different from $\left(K_{m} \pi_{m}\right)$ are not included in the un-normalized matrix elements of the calculation of Fig. 1.] Second, the truncation is effective of the two quasiparticle states used in the calculation of Eq. (2).

In summary, the formulation and the calculation of the overlap of the QRPA states have been shown. This is currently the most advanced calculation of the overlap, and it is important to investigate how this method affects the nuclear matrix elements in the QRPA; the preparation of the calculation is in progress. 


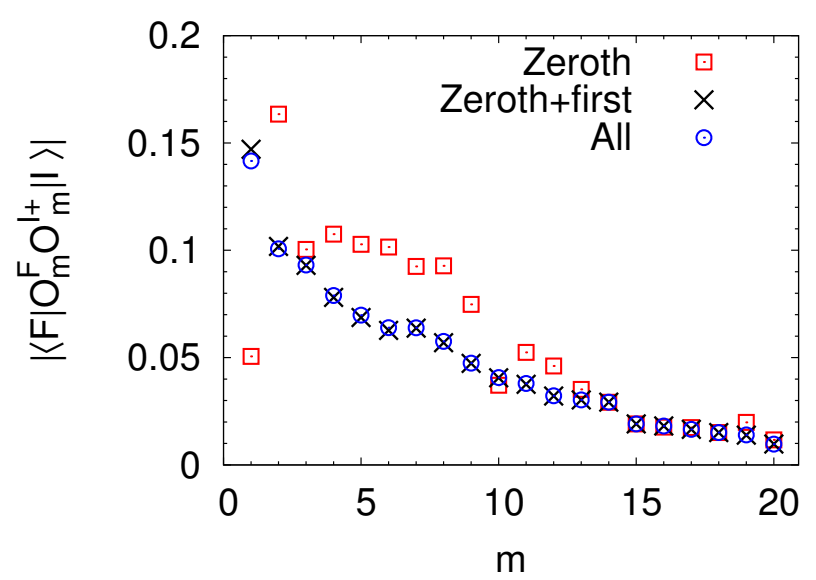

Fig. 1. Twenty largest absolute values of diagonal overlap matrix elements of ${ }^{26} \mathrm{Mg}$ $(|I\rangle)$ and ${ }^{26} \mathrm{Si}(|F\rangle)$. The zeroth (first) indicated in the panel refer to the first (second and third) term(s) of the right side of Eq. (2). 'All' in the panel indicates the result of the full calculation of Eq. (2). $(K \pi)$ of the QRPA states $m$ is $(0+)$.

This research is supported by the Ministry of Education, Culture, Sports, Science and Technology, Japan under the Grant-in-Aid for Scientific Research No. 23840005 and HPCI Strategic Program Field 5. The computers used for our calculations are those at the Center for Computational Sciences, University of Tsukuba, under the Collaborative Interdisciplinary Program and the Computational Fundamental Science Project (T2K-Tsukuba); the Yukawa Institute for Theoretical Physics, Kyoto University (SR16000); and the Research Center for Nuclear Physics, Osaka University (SX-8R).

\section{REFERENCES}

[1] M. Doi, T. Kotani, E. Takasugi, Prog. Theor. Phys. Suppl. 83, 1 (1985).

[2] J. Terasaki, arXiv:1208.3724 [nucl-th], to be published in Phys. Rev. C.

[3] A. Faessler, arXiv:1203.3648 [hep-ph].

[4] F. Šimkovic, G. Pantis, A. Faessler, Prog. Part. Nucl. Phys. 40, 285 (1998).

[5] J. Terasaki, Phys. Rev. C86, 021301 (2012).

[6] R. Balian, E. Brezin, Nuovo Cim. 64, 37 (1969).

[7] J. Bartel et al., Nucl. Phys. A386, 79 (1982).

[8] J. Dobaczewski et al., Phys. Rev. C53, 2809 (1996). 